\title{
Combining the multilevel fast multipole method with the uniform geometrical theory of diffraction
}

\section{A. Tzoulis and T. F. Eibert}

FGAN-Research Institute for High-Frequency Physics and Radar Techniques (FHR), 53343 Wachtberg-Werthhoven, Neuenahrer Str. 20, Germany

\begin{abstract}
The presence of arbitrarily shaped and electrically large objects in the same environment leads to hybridization of the Method of Moments (MoM) with the Uniform Geometrical Theory of Diffraction (UTD). The computation and memory complexity of the MoM solution is improved with the Multilevel Fast Multipole Method (MLFMM). By expanding the $\hat{k}$-space integrals in spherical harmonics, further considerable amount of memory can be saved without compromising accuracy and numerical speed. However, until now MoM-UTD hybrid methods are restricted to conventional MoM formulations only with Electric Field Integral Equation (EFIE). In this contribution, a MLFMM-UTD hybridization for Combined Field Integral Equation (CFIE) is proposed and applied within a hybrid Finite Element Boundary Integral (FEBI) technique. The MLFMM-UTD hybridization is performed at the translation procedure on the various levels of the MLFMM, using a far-field approximation of the corresponding translation operator. The formulation of this new hybrid technique is presented, as well as numerical results.
\end{abstract}

\section{Introduction}

Exact numerical solutions of electromagnetic radiation and scattering problems with composite dielectric/metallic objects are actually obtained with the Finite Element - Boundary Integral (FEBI) method. The involved integral equation (IE) is discretized by the Method of Moments (MoM) using Rao-Wilton-Glisson (RWG) basis functions and a Galerkin's type approach (Rao et al., 1982). The resulting hybrid linear equation system is efficiently solved with iterative solvers in combination with appropriate preconditioning (Eibert, 2003).

Computation complexity and memory requirements of large scale MoM solutions for high frequencies increase rapidly with the dimensions of the objects. This problem is overcome by the Multilevel Fast Multipole Method (MLFMM) resulting in low computation and memory complexity of $O(N \log N)$ (Coifman et al., 1993; Chew et al., 2001). Further saving of a considerable amount of memory

Correspondence to: A. Tzoulis (tzoulis@fgan.de) without compromising accuracy and numerical speed is achieved by expanding the FMM $\hat{k}$-space integrals into spherical harmonics (Eibert, 2004).

Electrically large conducting objects are handled efficiently with ray-based high-frequency methods. Such methods give accurate asymptotic solutions of the electromagnetic fields for objects with large dimensions as compared to the wavelength and no discretization is needed. The electromagnetic fields are described according to classical ray concepts of Geometrical Optics (GO) (Lo and Lee, 1993) and Uniform Geometrical Theory of Diffraction (UTD) (Kouyoumjian and Pathak, 1974).

Full electromagnetic coupling between dielectric arbitrarily shaped and UTD objects within a common environment is obtained with the hybrid FEBI-UTD method (Alaydrus et al., 2001), which is however restricted to conventional MoM formulations only with EFIE. In this contribution, the hybrid FEBI-UTD technique is extended by combining MLFMM with UTD for CFIE. The MLFMM-UTD hybridization is performed in the translation procedure on the various MLFMM levels. The amplitudes of the UTD rays are determined according to the far-field MLFMM in Chew et al. (2001). Due to different ray directions for outgoing and incoming waves, appropriate interpolation and anterpolation routines must be applied to consider the UTD contributions.

In the following, first the formulation of the FEBI-UTD for CFIE and after that the formulation of the MLFMM-UTD approach with expansion of the $\hat{k}$-space integrals in spherical harmonics is presented. Finally, numerical results for various large scale examples are given.

\section{Formulation}

\subsection{FEBI-UTD hybrid method}

Consider the configuration of Fig. 1, where inhomogeneous dielectric FEBI and large conducting UTD objects exist in the same environment in free space. The electromagnetic fields in the interior region of the FEBI objects are expressed according to the Finite Element Method (FEM) (Volakis et al., 1998) and are expanded in terms of edge element basis functions $\boldsymbol{\alpha}_{n}$ using tetrahedral volume meshes. 


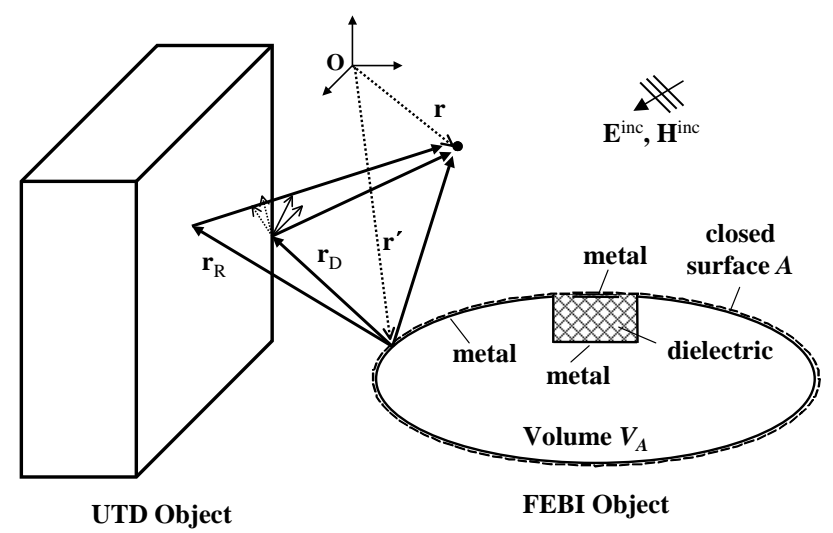

Fig. 1. Hybrid FEBI-UTD concept.

The fields in the exterior regions are expressed with IE's over the boundaries of the FEBI objects, being solved by a Galerkin-type MoM with RWG basis functions (Rao et al., 1982). The presence of UTD objects is taken into account by modifying the Green's functions, as well as the incident fields of the IE's, with additional high-frequency contributions received at the testing or observation points, respectively (Alaydrus et al., 2001). In this contribution, 1st and 2nd order reflections and diffractions on flat structures are taken into account. The following formulations will be given for clarity only for 1 st order mechanisms. The formulation for 2 nd order mechanisms can be easily given in the same manner.

The UTD ray contributions are taken into account for the CFIE

$Z(1-\alpha) M F I E-\alpha E F I E=0$,

where $\alpha$ is the combination parameter with values from 0 to 1 and $Z$ the wave impedance of the considered solution space. The EFIE is given by

$$
\begin{aligned}
\hat{n} \times[\hat{n} \times \quad & \left(\int \int \int _ { A } \left[\overline{\mathbf{G}}_{J, t o t}^{E}\left(\boldsymbol{r}, \boldsymbol{r}^{\prime}\right) \cdot \boldsymbol{J}_{A}\left(\boldsymbol{r}^{\prime}\right)\right.\right. \\
+ & \left.\left.\overline{\mathbf{G}}_{M, t o t}^{E}\left(\boldsymbol{r}, \boldsymbol{r}^{\prime}\right) \cdot \boldsymbol{M}_{A}\left(\boldsymbol{r}^{\prime}\right)\right] d a^{\prime}+\boldsymbol{E}_{t o t}^{i n c}(\boldsymbol{r})\right) \\
+ & \left.\frac{1}{2} \boldsymbol{M}_{A}(\boldsymbol{r})\right]=0
\end{aligned}
$$

and the Magnetic Field Integral Equation (MFIE) by

$$
\begin{aligned}
\hat{n} \times & \left(\int \int \int _ { A } \left[\overline{\mathbf{G}}_{M, t o t}^{H}\left(\boldsymbol{r}, \boldsymbol{r}^{\prime}\right) \cdot \boldsymbol{M}_{A}\left(\boldsymbol{r}^{\prime}\right)\right.\right. \\
+ & \left.\left.\overline{\mathbf{G}}_{J, t o t}^{H}\left(\boldsymbol{r}, \boldsymbol{r}^{\prime}\right) \cdot \boldsymbol{J}_{A}\left(\boldsymbol{r}^{\prime}\right)\right] d a^{\prime}+\boldsymbol{H}_{t o t}^{i n c}(\boldsymbol{r})\right) \\
& -\frac{1}{2} \boldsymbol{J}_{A}(\boldsymbol{r})=0,
\end{aligned}
$$

where $\overline{\mathbf{G}}_{J, t o t}^{E / H}\left(\boldsymbol{r}, \boldsymbol{r}^{\prime}\right)$ and $\overline{\mathbf{G}}_{M, t o t}^{E / H}\left(\boldsymbol{r}, \boldsymbol{r}^{\prime}\right)$ are the total Green's functions of the electric or magnetic field due to electric and

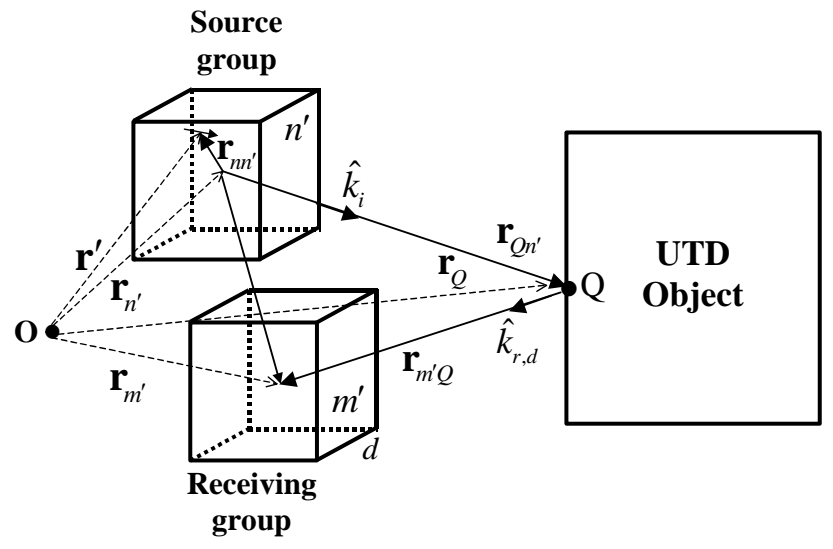

Fig. 2. Hybrid MLFMM-UTD concept.

magnetic surface currents, respectively. Also, $\boldsymbol{E}_{\text {tot }}^{i n c}(\boldsymbol{r})$ and $\boldsymbol{H}_{t o t}^{i n c}(\boldsymbol{r})$ are the total incident electric and magnetic fields at the observation point $\boldsymbol{r}$.

The total Green's functions of the hybrid problem are the superposition of the Green's functions of the direct coupling of the currents $\overline{\mathbf{G}}_{J / M}^{E / H}\left(\boldsymbol{r}, \boldsymbol{r}^{\prime}\right)$ and of

$$
\begin{aligned}
\overline{\mathbf{G}}_{J / M, U T D}^{E / H}\left(\boldsymbol{r}, \boldsymbol{r}^{\prime}\right) & =\sum_{s} A_{R_{s}} \overline{\mathbf{R}}_{s}^{E / H} \cdot \overline{\mathbf{G}}_{J / M}^{E / H}\left(\boldsymbol{r}_{R_{s}}, \boldsymbol{r}^{\prime}\right) \\
& +\sum_{v} A_{D_{v}} \overline{\mathbf{D}}_{v}^{E / H} \cdot \overline{\mathbf{G}}_{J / M}^{E / H}\left(\boldsymbol{r}_{D_{v}}, \boldsymbol{r}^{\prime}\right) \\
& +\cdots
\end{aligned}
$$

which is the Green's functions of the high-frequency raybased fields due to UTD objects. $A_{R_{s}}$ and $A_{D_{v}}$ are the divergence and phase factors for reflection and diffraction, respectively, and $\overline{\mathbf{R}}_{S}^{E / H}$ and $\overline{\mathbf{D}}_{v}^{E / H}$ the dyadic reflection and diffraction coefficients for electric or magnetic field defined by basic GO (Lo and Lee, 1993) and UTD concepts (Kouyoumjian and Pathak, 1974). Direct coupling is not taken into account if UTD objects lie between source and testing currents.

Similarly, the high-frequency contributions of the incident electric and magnetic field at the observation point $\boldsymbol{r}$ have the form

$$
\begin{aligned}
\boldsymbol{E}_{U T D}^{i n c}(\boldsymbol{r}) & =\sum_{s} A_{R_{s}} \overline{\mathbf{R}}_{s}^{E} \cdot \boldsymbol{E}^{i n c}\left(\boldsymbol{r}_{R_{s}}\right) \\
& +\sum_{v} A_{D_{v}} \overline{\mathbf{D}}_{v}^{E} \cdot \boldsymbol{E}^{i n c}\left(\boldsymbol{r}_{D_{v}}\right) \\
& +\cdots
\end{aligned}
$$

and

$$
\begin{aligned}
\boldsymbol{H}_{U T D}^{i n c}(\boldsymbol{r}) & =\frac{1}{Z} \hat{k}_{r} \times \sum_{s} A_{R_{s}} \overline{\mathbf{R}}_{s}^{E} \cdot \boldsymbol{E}^{i n c}\left(\boldsymbol{r}_{R_{s}}\right) \\
& +\frac{1}{Z} \hat{k}_{d} \times \sum_{v} A_{D_{v}} \overline{\mathbf{D}}_{v}^{E} \cdot \boldsymbol{E}^{i n c}\left(\boldsymbol{r}_{D_{v}}\right) \\
& +\cdots,
\end{aligned}
$$




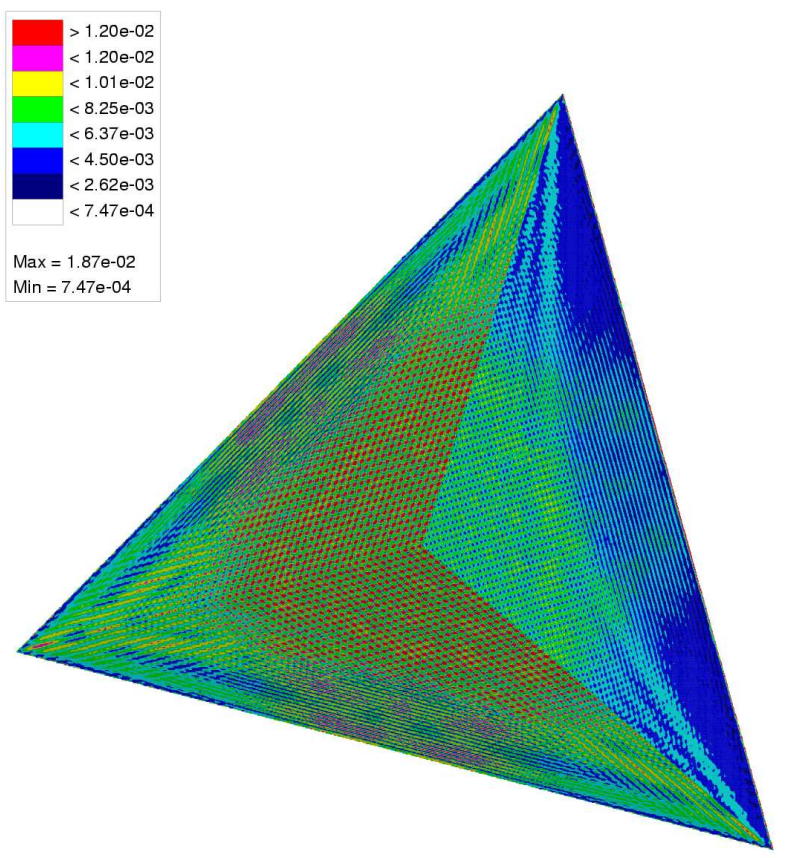

Fig. 3. Surface current density magnitude of trihedral $90^{\circ}$ corner reflector for $15 \mathrm{GHz}$.

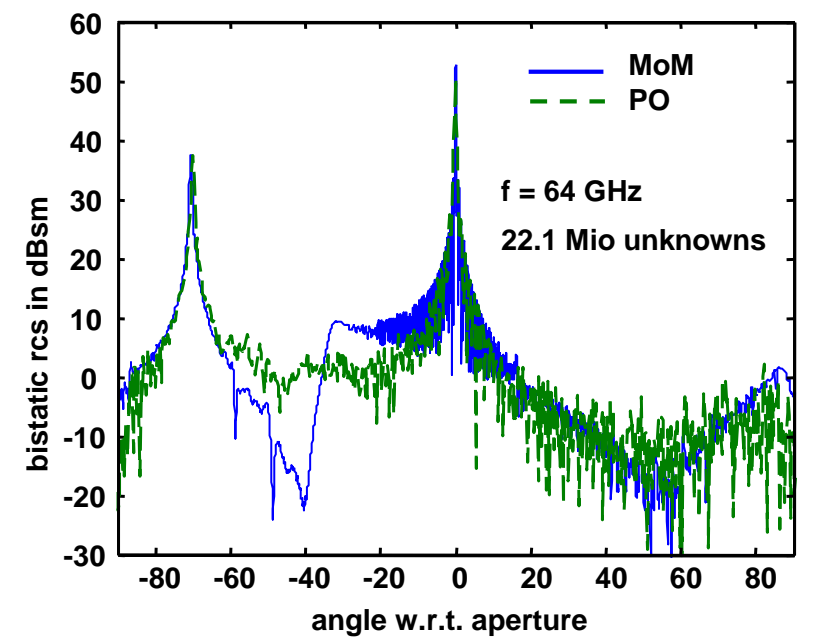

Fig. 4. Bistatic RCS of trihedral $90^{\circ}$ corner reflector for $64 \mathrm{GHz}$.

where $\hat{k}_{r}$ and $\hat{k}_{d}$ are the propagation directions of the reflected and diffracted rays, respectively. Again, no direct field contribution is taken into account if the observation point is in the shadow of an UTD object.

The MoM solution of the IE's (1), (2) and (3) is achieved by expanding the surface currents in terms of RWG basis functions $\boldsymbol{\beta}_{n}$ using triangular surface meshes. The exterior fields are coupled with the interior ones via field continuity conditions at the boundaries of the FEBI objects. The resulting hybrid linear equation system is solved by an iterative solution technique, using a multilevel iterative preconditioning strategy (Eibert, 2003).
The matrix-vector product computations are accelerated by MLFMM. In order to retain low complexity in the hybrid approach, UTD contributions are taken into account in the matrix-vector product MLFMM computations. In the following, the MLFMM formulation for CFIE with expansion of the $\hat{k}$-space integrals in spherical harmonics, as well as the hybrid MLFMM-UTD formulation is presented.

\subsection{MLFMM-UTD hybrid method}

The hybrid MLFMM-UTD concept is shown in Fig. 2. The source group $n^{\prime}$ and the receiver group $m^{\prime}$ belong to the FMM model of the FEBI object at a specific level. The CFIE matrix elements in FMM representation can be written as (Coifman et al., 1993; Chew et al., 2001)

$$
\begin{aligned}
& Z_{m n, J}^{C F I E}= \\
& -c_{1} \oiint \tilde{\boldsymbol{\beta}}_{m}^{*}(\hat{k}) \cdot T_{L}\left(\hat{k} \cdot \hat{r}_{m^{\prime} n^{\prime}}\right)(\overline{\mathbf{I}}-\hat{k} \hat{k}) \cdot \tilde{\boldsymbol{\beta}}_{n}(\hat{k}) d \hat{k}^{2} \\
& +c_{2} \oiint\left(\hat{k} \times \tilde{\boldsymbol{\alpha}}_{m}^{*}(\hat{k})\right) \cdot T_{L}\left(\hat{k} \cdot \hat{r}_{m^{\prime} n^{\prime}}\right) \tilde{\boldsymbol{\beta}}_{n}(\hat{k}) d \hat{k}^{2}
\end{aligned}
$$

and

$$
\begin{aligned}
& Z_{m n, M}^{C F I E}= \\
& +c_{3} \oiint\left(\hat{k} \times \tilde{\boldsymbol{\beta}}_{m}^{*}(\hat{k})\right) \cdot T_{L}\left(\hat{k} \cdot \hat{r}_{m^{\prime} n^{\prime}}\right) \tilde{\boldsymbol{\beta}}_{n}(\hat{k}) d \hat{k}^{2} \\
& +c_{4} \oiint \tilde{\boldsymbol{\alpha}}_{m}^{*}(\hat{k}) \cdot T_{L}\left(\hat{k} \cdot \hat{r}_{m^{\prime} n^{\prime}}\right)(\overline{\mathbf{I}}-\hat{k} \hat{k}) \cdot \tilde{\boldsymbol{\beta}}_{n}(\hat{k}) d \hat{k}^{2}
\end{aligned}
$$

where $c_{1}=j \frac{\omega \mu}{4 \pi} \alpha, c_{2}=j Z_{0} \frac{k}{4 \pi}(1-\alpha), c_{3}=j \frac{k}{4 \pi} \alpha$ and $c_{4}=j Z_{0} \frac{\omega \varepsilon}{4 \pi}(1-\alpha)$. Further,

$\tilde{\boldsymbol{\beta}}_{m}(\hat{k})=\iint_{A} \boldsymbol{\beta}_{m}(\boldsymbol{r}) e^{j \boldsymbol{k} \cdot \boldsymbol{r}_{m m^{\prime}}} d a$

and

$$
\tilde{\boldsymbol{\alpha}}_{m}(\hat{k})=\iint_{A} \boldsymbol{\alpha}_{m}(\boldsymbol{r}) e^{j \boldsymbol{k} \cdot \boldsymbol{r}_{m m^{\prime}}} d a
$$

are the $\hat{k}$-space representations of the basis functions and

$T_{L}(\hat{k} \cdot \hat{r})=\sum_{l=0}^{L}(-j)^{l}(2 l+1) \mathrm{h}_{l}^{(2)}(k r) \mathrm{P}_{l}(\hat{k} \cdot \hat{r})$

is the translation operator. Also, $\mathrm{h}_{l}^{(2)}$ is the second kind spherical Hankel function of degree $l, \mathrm{P}_{l}$ is the Legendre polynomial of degree $l$ and $*$ denotes complex conjugation. The MLFMM is implemented with memory efficiency by expanding the representations $\tilde{\boldsymbol{\beta}}_{m}(\hat{k})$ of the basis functions in spherical harmonics according to

$\tilde{\boldsymbol{\beta}}_{m}(\hat{k})=\sum_{p=0}^{P} \sum_{q=-p}^{p} \boldsymbol{f}_{p q}^{n} \mathrm{Y}_{p q}(\vartheta, \varphi)$, 


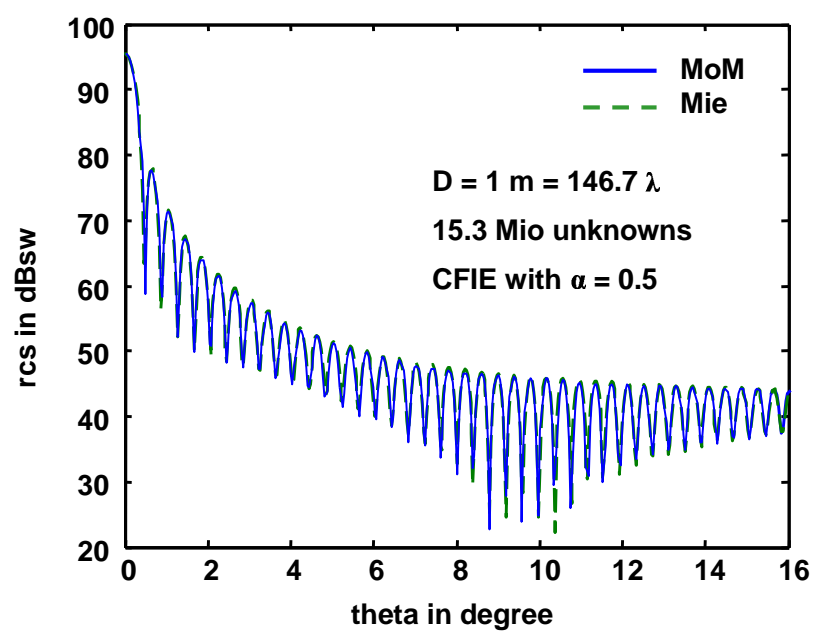

Fig. 5. RCS near forward direction of conducting sphere with diameter $D=1 \mathrm{~m}=146.7 \lambda$ by CFIE solution.

where

$\mathrm{Y}_{p q}(\vartheta, \varphi)=\sqrt{\frac{(2 p+1)(p-q) !}{4 \pi(p+q) !}} \mathrm{P}_{p}^{q}(\cos \vartheta) e^{j q \varphi}$,

are the orthonormalized spherical harmonics (Harrington, 1961). Also, $\mathrm{P}_{p}^{q}$ is the associated Legendre polynomial of degree $p$ and order $q$. According to this approach, the matrixvector product computations within the iteration loop of the iterative solver are performed by first aggregating all expansion coefficients at the center of each MLFMM group at the finest level. After that, the outgoing waves are computed at the quadrature points for all groups on the finest MLFMM level. The translations of outgoing waves into incoming waves as well as the aggregations and disaggregations between different MLFMM levels including interpolation and anterpolation are performed as in standard MLFMM using the numerical quadrature samples. However, when all incoming waves are collected in a certain group on the finest level, the spherical harmonics expansion

$T_{L}\left(\hat{k} \cdot \hat{r}_{m^{\prime} n^{\prime}}\right)(\overline{\mathbf{I}}-\hat{k} \hat{k}) \cdot \tilde{\boldsymbol{\beta}}_{n}(\hat{k})=\sum_{p=0}^{P} \sum_{q=-p}^{p} \boldsymbol{g}_{p q}^{n} \mathrm{Y}_{p q}(\vartheta, \varphi)$

is carried out and by utilizing the orthogonality of the spherical harmonics, the closed integral over the Ewald sphere is simplified to the series

$Z_{m n}=-j \frac{\omega \mu}{4 \pi} \sum_{p=0}^{P} \sum_{q=-p}^{p}\left(\boldsymbol{f}_{p q}^{m}\right)^{*} \cdot \boldsymbol{g}_{p q}^{n}$.

The number of expansion coefficients needed with this approach is in general less than the corresponding number of quadrature samples for numerical integration in the $\hat{k}$-space, resulting in saving of memory requirements.

The ray contributions due to UTD objects are taken into account in the MLFMM matrix-vector product computations

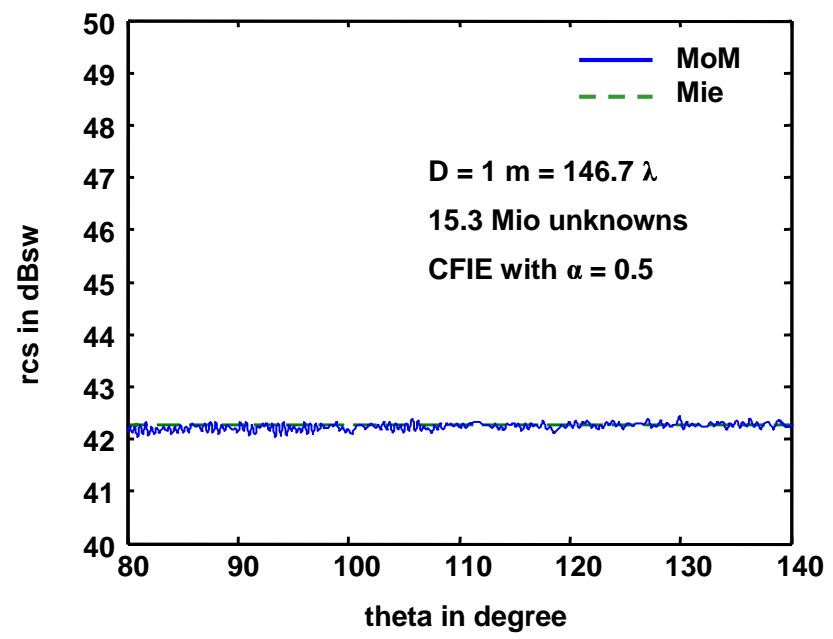

Fig. 6. RCS near backward direction of conducting sphere with diameter $D=1 \mathrm{~m}=146.7 \lambda$ by CFIE solution.

by assuming that the ray path from the source group to the UTD object $\boldsymbol{r}_{Q n^{\prime}}$ is much greater than the dimensions of the group itself. This means, that only one $\hat{k}$ direction is needed to express the radiated field from the source group. Assuming far-field conditions, the scalar Green's function from the source current to the local point on the UTD object is expressed with the far-field MLFMM approximation

$G\left(\boldsymbol{r}_{Q}, \boldsymbol{r}^{\prime}\right)=\frac{e^{-j k\left|\boldsymbol{r}_{Q}-\boldsymbol{r}^{\prime}\right|}}{\left|\boldsymbol{r}_{Q}-\boldsymbol{r}^{\prime}\right|}=e^{j \boldsymbol{k}_{i} \cdot \boldsymbol{r}_{n n^{\prime}}} T_{L}^{F F}$,

where $\boldsymbol{k}_{i}=k \hat{k}_{i}, \hat{k}_{i}=\hat{r}_{Q n^{\prime}}$ is the direction of ray incidence and

$T_{L}^{F F}=\frac{e^{-j k r_{Q n^{\prime}}}}{r_{Q n^{\prime}}}$

is the associated far-field translation operator (Chew et al., 2001). Thus, the translation of the high-frequency contributions is performed only for the direction of ray incidence $\hat{k}_{i}$. The received high-frequency fields are taken into account only for the reflection $\hat{k}_{r}$ or diffraction $\hat{k}_{d}$ directions from the local point on the UTD object to the receiver groups. The following MLFMM-UTD formulations are given for simplicity for the case of the EFIE contributions in (7). However, they apply to all contributions in (7) and (8) as well. The ray contributions of the matrix elements of the hybrid MLFMMUTD approach can be written as

$$
\begin{aligned}
& Z_{m n, J, U T D}^{E F I E}= \\
& -j \frac{\omega \mu}{4 \pi} \tilde{\boldsymbol{\beta}}_{m}^{*}\left(\hat{k}_{r}\right) \cdot \sum_{s} A_{R_{s}} \overline{\mathbf{R}}_{s}^{E} T_{L}^{F F} \cdot\left(\overline{\mathbf{I}}-\hat{k}_{r} \hat{k}_{i}\right) \cdot \tilde{\boldsymbol{\beta}}_{n}\left(\hat{k}_{i}\right) \\
& -j \frac{\omega \mu}{4 \pi} \tilde{\boldsymbol{\beta}}_{m}^{*}\left(\hat{k}_{d}\right) \cdot \sum_{v} A_{D_{v}} \overline{\mathbf{D}}_{v}^{E} T_{L}^{F F} \cdot\left(\overline{\mathbf{I}}-\hat{k}_{d} \hat{k}_{i}\right) \cdot \tilde{\boldsymbol{\beta}}_{n}\left(\hat{k}_{i}\right) \\
& +\cdots,
\end{aligned}
$$

where $\boldsymbol{k}_{r}=k \hat{k}_{r}$ and $\boldsymbol{k}_{d}=k \hat{k}_{d}$. Again, no direct field contribution is taken into account if the receiver group is in the shadow of an UTD object. 


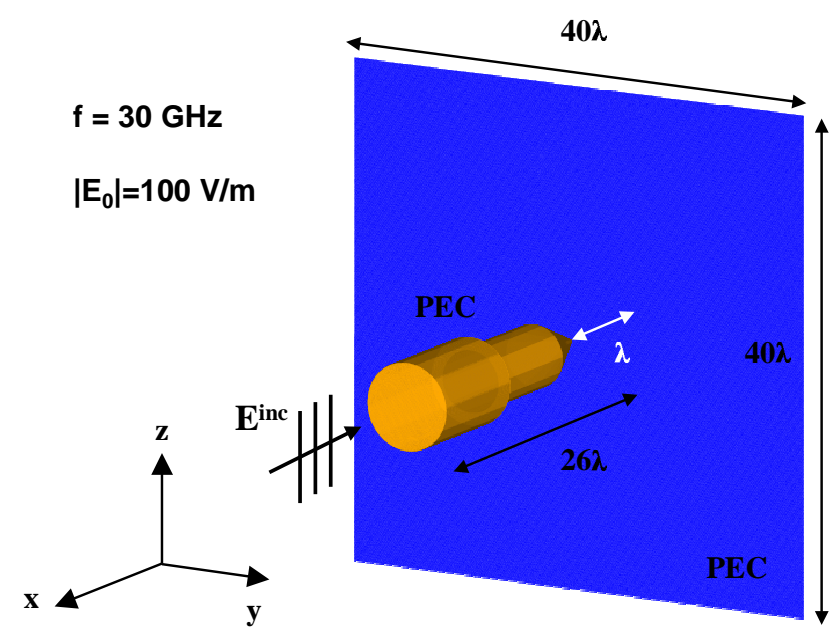

Fig. 7. Conducting cone cylinder in front of conducting plate at distance $\lambda$.

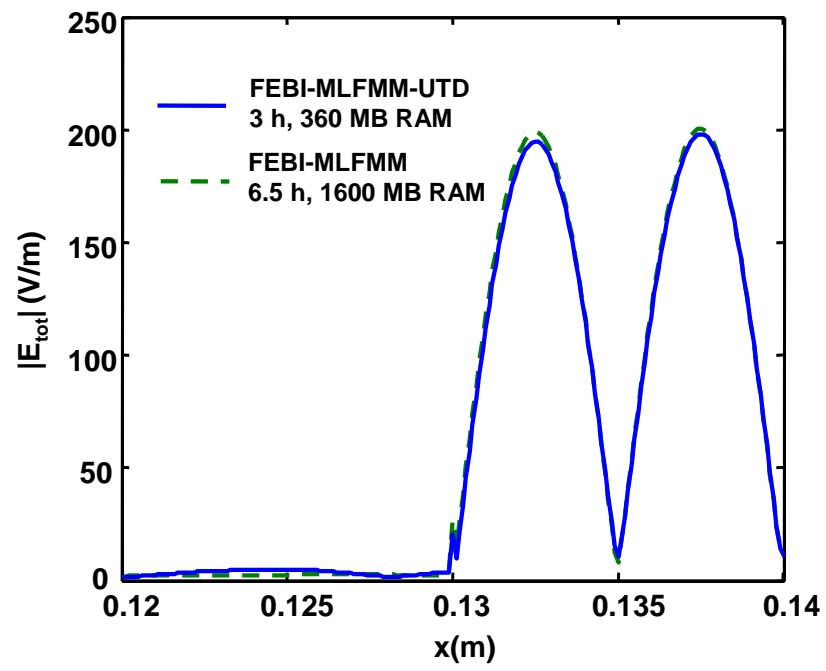

Fig. 8. Electric field in direction of propagation for conducting cone cylinder in front of conducting plate.

In the numerical implementation of MLFMM, a limited number of sampling points is used to evaluate the $\hat{k}$-space integrals. In general, the ray directions of incidence $\hat{k}_{i}$, reflection $\hat{k}_{r}$ or diffraction $\hat{k}_{d}$ do not match with any of these sampling points. For this reason, the required direction of incidence must be interpolated from the neighboring sampling points. Similarly, after reflection or diffraction, the appropriate ray direction must be anterpolated to the neighboring sampling points.

\section{Numerical examples}

In this section, numerical results of various large scale examples are presented. First, a trihedral $90^{\circ}$ conducting corner reflector with $1.4 \mathrm{~m}$ edge length has been computed, as

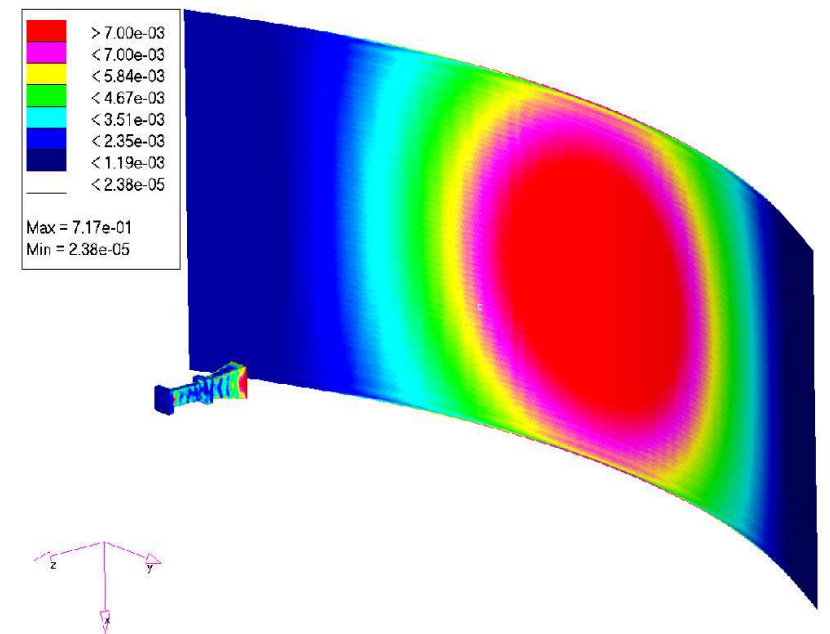

Fig. 9. Magnitude of surface current density on conducting cylindrical parabolic reflector with X-band horn excitation for $f=10 \mathrm{GHz}$.

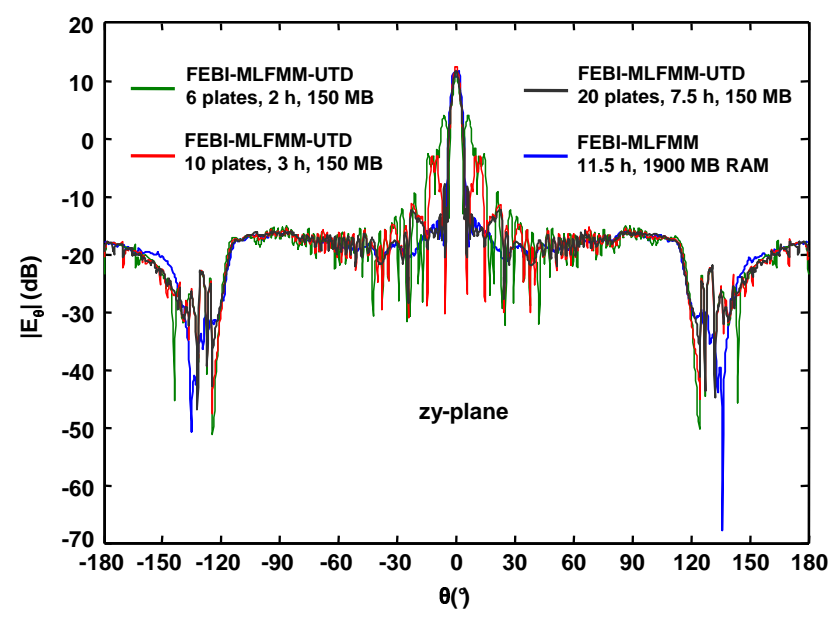

Fig. 10. Co-polar radiation pattern on principal E-plane of conducting cylindrical parabolic reflector with $\mathrm{X}$-band horn excitation for $f=10 \mathrm{GHz}$.

shown in Fig. 3. In the same figure, the magnitude of the surface current density on the corner reflector can be seen, computed for $15 \mathrm{GHz}$, using a triangular surface mesh. For the same corner reflector, RCS computations have been carried out for $64 \mathrm{GHz}$, using $\mathrm{H}$-polarized plane wave excitation with normal incidence. In this case, the reflector was discretized with 22.1 Million unknowns and the computation time of the solution was about $122 \mathrm{~h}$ on an Opteron $2.2 \mathrm{GHz}$ CPU with about $28 \mathrm{~GB}$ memory requirements. In Fig. 4, the co-polar bistatic RCS can be seen, compared to a PO solution. It is noticed, that to our knowledge this computation is currently the world-wide largest MoM solution performed on a single CPU.

Next example is a conducting sphere with diameter $D=1 \mathrm{~m}=146.7 \lambda$. The triangular surface mesh on the sphere resulted in 15.3 Million unknowns and the solution was 
obtained with FEBI for CFIE with $\alpha=0.5$. In Figs. 5 and 6, the computed RCS is shown, for small regions near forward and backward direction, respectively. The numerical results are compared to the corresponding Mie-series analytical solution. It can be seen, that numerical results have excellent agreement with the exact analytical solution. Especially at backward direction, very small ripple of the numerical solution can be seen.

Next example is the problem of a conducting cone cylinder in front of a conducting plate at distance $\lambda$, as shown in Fig. 7. Two computations have been carried out. First, both objects where discretized with triangular surface mesh, resulting in 630628 unknowns. In the second computation, the same surface mesh was used for the cone cylinder and the plate was treated by UTD. In this case, the number of unknowns was 150492 . In Fig. 8, the calculated electric field in direction of propagation for both computations is shown. In the same figure, computation time and memory requirements on an AMD Athlon 2000+ PC are shown as well. It can be seen, that the hybrid FEBI-UTD results agree very well with the exact numerical FEBI solution, needing much less computation time and memory requirements.

Last example is a conducting cylindrical parabolic reflector with X-band horn excitation for $f=10 \mathrm{GHz}$, as shown in Fig. 9. In the same figure, the magnitude of the surface current density on both objects can be seen, using a triangular surface mesh for both objects with 830280 unknowns. For the hybrid FEBI-UTD computation, the reflector was approximated by flat plates and computations were done for 6 , 10 and 20 plates. In Fig. 10, the co-polar radiation pattern on the principal E-plane for all computations is shown. In the same figure, the computation time and memory requirements on an AMD Athlon 2000+ PC are shown as well. It can be seen, that for 6 and 10 plates the hybrid FEBI-UTD solution presents significant side lobes. For 20 plates, the solution shows very good agreement with the exact numerical FEBI solution, needing less computation time and memory requirements.

\section{Conclusions}

In this contribution, the hybrid FEBI-MLFMM-UTD method for numerical radiation and scattering computations was presented. Due to memory efficient implementation of the MLFMM, large scale numerical computations can be carried out. The FEBI-UTD hybridization allows optimum treatment of problems including arbitrarily shaped composite dielectric/metallic objects and electrically large conducting objects of simple shape in the same environment. Low computation complexity and memory requirements are retained in the hybrid FEBI-UTD approach, due to MLFMM-UTD combination. The MLFMM-UTD hybridization is performed in the translation procedure on the various MLFMM levels, using a far-field approximation of the appropriate translation operator. The hybrid FEBI-UTD formulation for CFIE, as well as the formulation of the MLFMM-UTD combination with expansion of the $\hat{k}$-space integrals in spherical harmonics was presented. Finally, numerical results for various large scale antenna and scattering problems were given.

\section{References}

Alaydrus, M., Hansen, V., and Eibert, T. F.: Hybrid ${ }^{2}$ : Combining the three-dimensional hybrid finite element - boundary integral technique for planar multilayered media with the uniform geometrical theory of diffraction, IEEE Trans. Antennas Propagat., vol. 50, no. 1, 67-74, Jan. 2002.

Chew, W. C., Jin, J.-M., and Michielssen, E.: Fast and efficient algorithms in computational electromagnetics, Boston: Artech House, 2001.

Chew, W. C., Cui, T. J., and Song, J. M.: A FAFFA-MLFMA algorithm for electromagnetic scattering, IEEE Trans. Antennas Propagat., vol. 50, no. 11, 1641-1649, Nov. 2002.

Coifman, R., Rokhlin, V., and Wandzura, S.: The fast multipole method: A pedestrian prescription, IEEE Antennas Propagat. Mag., vol. 35, no. 3, 7-12, Jun. 1993.

Eibert, T. F.: Advances in hybrid finite element - boundary integral modelling of three-dimensional radiation and scattering problems, Intern. ITG Conf. on Antennas, 157-160, Berlin, Sep. 2003.

Eibert, T. F.: A multilevel fast multipole method with spherical harmonics expansion of the $k$-space integrals, URSI EM-Theory Symposium, Pisa, Italy, 150-153, May 2004.

Harrington, R. F.: Time harmonic electromagnetic fields, New York: McGraw-Hill, 1961.

Kouyoumjian, R. G. and Pathak, P. R.: A uniform geometrical theory of diffraction for an edge in a perfectly conducting surface, Proc. IEEE, vol. 62, no. 11, 1448-1461, Nov. 1974.

Lo, Y. T. and Lee, S. W.: Antenna handbook, vol.1, Chapman \& Hall, New York, 1993.

Rao, S. M., Wilton, D. R., and Glisson, A. W.: Electromagnetic scattering by surfaces of arbitrary shape, IEEE Trans. AP, vol. 30, 409-418, May 1982.

Volakis, J. L., Chatterjee, A., and Kempel, L.-C.: Finite Element Method for Electromagnetics, IEEE, Inc., New York, 1998. 